\title{
COLOR IMAGE PROCESSING USING GENERALIZED WEIGHTED VECTOR FILTERS
}

Rastislav Lukac, Konstantinos N. Plataniotis, Anastasios N. Venetsanopoulos The Edward S. Rogers Sr. Department ECE

University of Toronto, 10 King's College Road

Toronto, M5S 3 G4 Ontario, Canada

lukacr@ieee.org, kostas@dsp.utoronto.ca, anv@dsp.utoronto.ca

\author{
Bogdan Smolka* \\ Polish-Japanese Institute of Information Technology, \\ Koszykowa 86 Str, 02-008 Warsaw, Poland \\ bsmolka@ia.polsl.gliwice.pl
}

\begin{abstract}
This paper focuses on generalized weighted vector filters which constitute a powerful filtering frameworkfor multichannel signal processing. The utilization of a generalized multichannel sigmoidal adaptation scheme allows to accommodate the filter parameters to varying signal and noise statistics. Experimental results indicate that the presented multichannel filters are capable of removing impulsive noise present in color images and simultaneously they preserve color and structural information.
\end{abstract}

Keywords: Multichannel image processing; color image filtering; filter weights; adaptation algorithm.

\section{INTRODUCTION}

In the last decade, a number of nonlinear multichannel filters have been developed $^{1-3}$. Among them, vector filters take a great interest of the image processing community due to the importance of color image processing ${ }^{4}$. It is well-known that noise and other impairments introduced during recording and/or transmission of the image data degrade the value of the color information. This usually declines the perceptual quality and the fidelity of the images, and decreases the performance of the task for which the image was used ${ }^{5}$.

*This research has been supported by a grant No PJ/B/01/2004 from the Polish - Japanese Institute of Information Technology. 
Therefore, noise filtering is an essential part of any image processing based system. In this paper, we focus on impulsive noise removal in color images using generalized weighted vector filters ${ }^{5}$.

\section{GENERALIZED WEIGHTED VECTOR FILTERS}

Let us consider, a $K_{1} \times K_{2}$ multichannel image $\mathbf{x}: Z^{l} \rightarrow Z^{m}$ representing a two-dimensional matrix of $m$-component samples $\mathbf{x}_{i}=\left[x_{i 1}, x_{i 2}, \ldots, x_{i m}\right]$. Note that standard color images such as Red-Green-Blue (RGB) images relate to parameters $l=2$ and $m=3$. Components $x_{i k}$, for $k=1,2, \ldots, m$ and $i=1,2, \ldots, K_{1} K_{2}$, represent the $k$-th elements of the vectorial inputs $\mathbf{x}_{i}$. Thus, each multichannel sample $\mathbf{x}_{i}$ can be considered as a $m$-dimensional vector. Operating on some type of sliding window $W=\left\{\mathbf{x}_{i} \in Z^{l} ; i=1,2, \ldots, N\right\}$ of finite size $N$, the window center $\mathbf{x}_{(N+1) / 2}$ is replaced by some function of a local neighborhood area $W$. This window operator slides over the image to affect individually all the image pixels.

It has been widely observed that vector processing is the most effective way to process noisy color images ${ }^{1-5}$. Since the noisy samples usually represent extremes of the input set, they can be effectively determined using the aggregated distance concept applied to the samples of $W$. The extreme observations maximize the aggregated distances to other samples of $W$ and therefore, the most popular vector filters determine the output as the lowest ranked vector in a predefined sliding window ${ }^{4,5}$. The well-known vector median filters $(\mathrm{VMF})^{1}$ utilize the aggregated Euclidean distance, whereas vector directional filters $(\mathrm{VDF})^{2}$ utilize the aggregated angular distance of the input vectors.

Employing the concept of generalized weighted vector filters $(\mathrm{WVF})^{5}:$ i) a number of previously developed vector filters (including VMF andVDF) are generalized, ii) more sophisticated filtering solutions can be designed, and iii) the characteristics of the solution can be adapt for various image and noise statistics. In the WVF design, the vectors $\mathbf{w}=\left[w_{1}, w_{2}, \ldots, w_{N}\right]$ and $\mathbf{u}=$ $\left[u_{1}, u_{2}, \ldots, u_{N}\right]$ represent sets of positive real weights reflecting the magnitude and directional characteristics of the input samples $\mathbf{x}_{i}$ associated with the weights $w_{i}$ and $u_{i}$, for $i=1,2, \ldots, N$. Using the minimization principle, the WVF scheme is defined as follows ${ }^{5}$ :

$$
\min \underset{\mathbf{x}_{(1)} \in W}{\arg }\left(\sum_{i=1}^{N} w_{i}\left\|\mathbf{x}_{(1)}-\mathbf{x}_{i}\right\|_{2}\right)^{1-p}\left(\sum_{i=1}^{N} u_{i} A\left(\mathbf{x}_{(1)}, \mathbf{x}_{i}\right)\right)^{p}
$$

where $\left\|\mathbf{x}_{(1)}-\mathbf{x}_{i}\right\|_{2}$ is the Euclidean distance, whereas $A\left(\mathbf{x}_{(1)}, \mathbf{x}_{i}\right)$ denotes the angular distance between two color vectors $\mathbf{x}_{(1)}$ and $\mathbf{x}_{i}$. The parameter $p$ is a design parameter ranged from 0 to 1 and it can be used to tune the overallfilter characteristics.

To adapt the WVF coefficients in required manner, a multichannel generalization of the sigmoidal adaptation scheme of ${ }^{6}$ has been provided ${ }^{5}$. Assu- 
ming, for the simplicity, the equivalence between the weight vectors $\mathbf{w}$ and $\mathbf{u}$ so that $w_{i}=u_{i}$, for $i=1,2, \ldots, N$, the WVF can be defined using $\mathbf{w}=$ $\left[w_{1}, w_{2}, \ldots, w_{N}\right]$, only. Employing the vector approach of, ${ }^{5}$ the generalized difference between two vectors $\mathbf{x}_{i}$ and $\mathbf{x}_{j}$ is defined as follows:

$$
D\left(\mathbf{x}_{i}, \mathbf{x}_{j}\right)=S\left(\mathbf{x}_{i}, \mathbf{x}_{j}\right)\left(\left\|\mathbf{x}_{i}-\mathbf{x}_{j}\right\|_{2}\right)^{1-p}\left(A\left(\mathbf{x}_{i}, \mathbf{x}_{j}\right)\right)^{p}
$$

where $S\left(\mathbf{x}_{i}, \mathbf{x}_{j}\right) \in\{-1,1\}$ is a polarity function given by $S\left(\mathbf{x}_{i}, \mathbf{x}_{j}\right)=1$ for $\left\|\mathbf{x}_{i}\right\| \geq\left\|\mathbf{x}_{j}\right\|$, and $S\left(\mathbf{x}_{i}, \mathbf{x}_{j}\right)=-1$ for $\left\|\mathbf{x}_{i}\right\|<\left\|\mathbf{x}_{j}\right\|$. The use of $S(\cdot, \cdot)$ preserves the sign of the difference between the scalar image samples $\mathbf{x}_{i}$ and $\mathbf{x}_{j}$, since for a scalar case, i.e. $m=1$ and $p=0$, the magnitude of $\mathbf{x}_{i}$ and $\mathbf{x}_{j}$ is equivalent to $x_{i 1}$ and $x_{j 1}$, respectively.

During processing, the sliding filtering window is moving over an image domain. With respect to the filter weights at the previous points, the iterative algorithm adjusts the weights $w_{i}$, for $i=1,2, \ldots, N$, as follows ${ }^{5}$ :

$$
w_{i}=P\left[w_{i}+2 \mu D(\mathbf{o}, \mathbf{y}) \operatorname{sgn}\left(D\left(\mathbf{x}_{i}, \mathbf{y}\right)\right)\right]
$$

where $\mathbf{o}$ denotes the desired (original) signal, $\mu$ is the regularization factor, $\mathbf{y}$ is the output of the sigmoidally optimized WVF filter (1) related to the actual weight coefficients $\mathbf{w}, \operatorname{sgn}(a)=\left(2 /\left(1+e^{-a}\right)\right)-1$ denotes the sign sigmoidal function and $P(\cdot)$ is a projection function which changes the negative values to zero during the weight adaptation. The initial weight vector $\mathbf{w}$ should contain the equal weights corresponding to the robust smoothing functions ${ }^{5}$.

Since, original noise-free (training) samples may not be available in practical image processing applications, the desired signal $o$ in (3) should be replaced with the robust, easily achieved estimate $\mathbf{y}^{*}$. Using a component-wise median filter (MF), ${ }^{7}$ the adaptation formula (3) is modified as follows ${ }^{5}$ :

$$
w_{i}=P\left[w_{i}+2 \mu D\left(\mathbf{y}^{*}, \mathbf{y}\right) \operatorname{sgn}\left(D\left(\mathbf{x}_{i}, \mathbf{y}\right)\right)\right]
$$

where $\mathbf{y}^{*}=\left[y_{1}^{*}, y_{2}^{*}, \ldots, y_{m}^{*}\right]$ is the MF of the input set $W$.

To improve the detail-preserving characteristics of (4), it can be desirable to simultaneously use the MF output $\mathbf{y}^{*}$ and the central sample $\mathbf{x}_{(N+1) / 2},{ }^{5}$ :

$$
w_{i}=P\left[w_{i}+2 \mu\left(D\left(\mathbf{y}^{*}, \mathbf{y}\right)+D\left(\mathbf{x}_{(N+1) / 2}, \mathbf{y}\right)\right) \operatorname{sgn}\left(D\left(\mathbf{x}_{i}, \mathbf{y}\right)\right)\right]
$$

Such a filter should demonstrate the excellent balance noise smoothing and color/structural content preservation.

\section{EXPERIMENTAL RESULTS}

The adaptation capability of the WVF framework is tested using $256 \times 256$ test color image Lena (Fig.1a,b). In all subsequent experiments a $3 \times 3$ supporting window (i.e. $N=9$ ) is used. The results are measured using the 


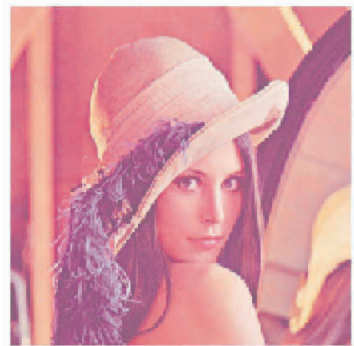

(a)

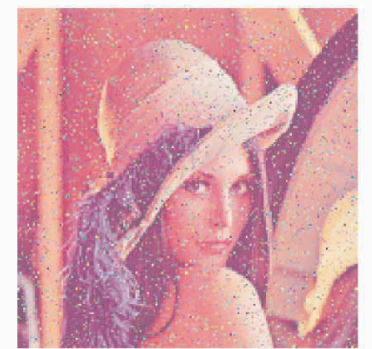

(b)

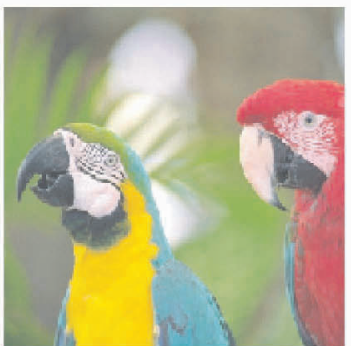

(c)

Figure 1. $256 \times 256$ test color images: (a) original image Lena, (b) image Lena corrupted by $10 \%$ impulsive noise, (c) original image Parrots.
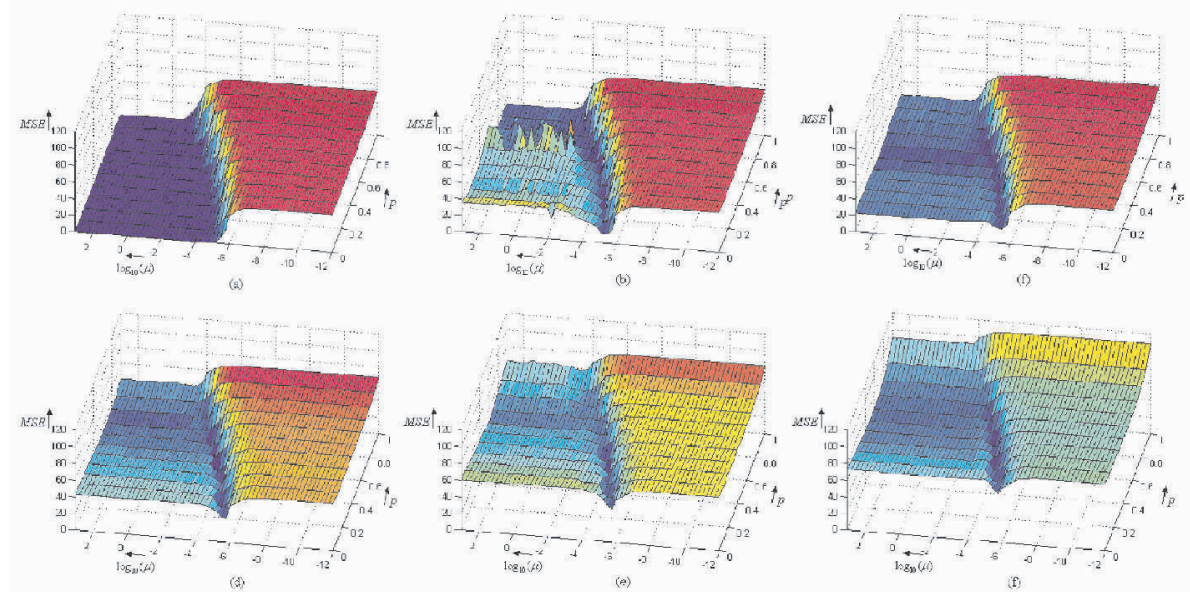

Figure 2. WVF adaptation capability v.s. MSE expressed for the image Lena corrupted by impulsive noise: (a) $0 \%$ noise, (b) $2 \%$ noise, (c) $5 \%$ noise, (d) $10 \%$ noise, (e) $15 \%$ noise, (f) $20 \%$ noise.

commonly accepted mean square error (MSE) and normalized color difference (NCD) criteria $^{4,5}$. Fig. 2 shows the results, expressed in terms of MSE, are related to the training set represented by the test image Lena and its corrupted versions with $0 \%$ (no corruption), 2\%, 5\%, 10\%, 15\% and 20\% impulsive noise, respectively. It can be seen that the error criteria increase with the noise corruption and that in all cases the WVF scheme achieved the best results for $\mu \geq 0.5$.

The WVF filters optimized using the image Lena corrupted by $10 \%$ impulsive noise are compared, in terms of performance related to the test color image 


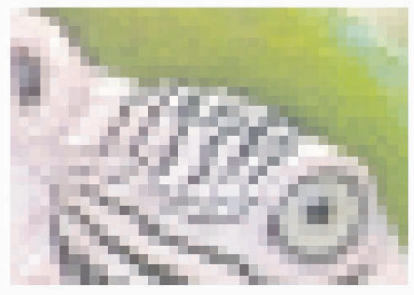

(a)

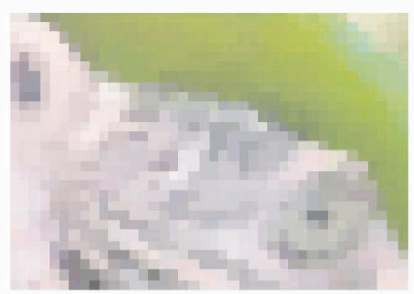

(c)

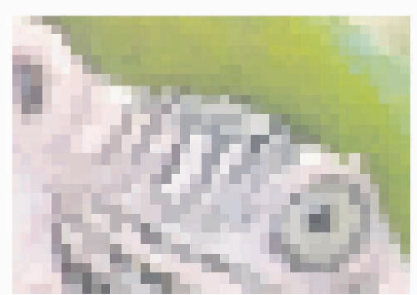

(e)

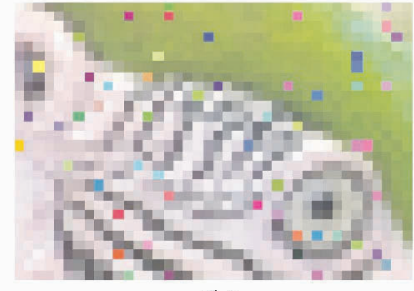

(b)

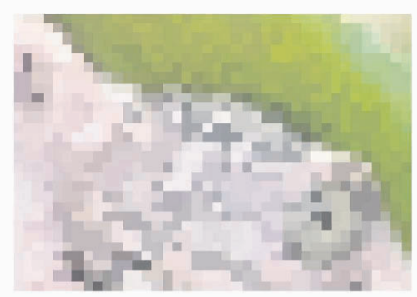

(d)

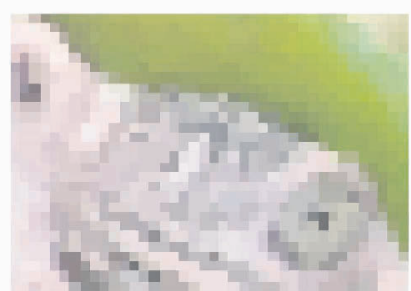

(f)

Figure 3. Enlarged parts of Parrots image: (a) original, (b) image corrupted by 5\% impulsive noise, (c) VMF output, (d) BVDF output, (e) WVF output obtained via (3), (f) WVF output obtained via (5).

Parrots (Fig.1c) with other widely used color image filters such as VMF, ${ }^{1}$ basic VDF (BVDF) ${ }^{2} \mathrm{MF}^{7}$ and directional-distance filter (DDF) ${ }^{8}$.

Visual inspection of the results shown in Table 1 and Fig.3 reveals that the WVF schemes achieve excellent balance between noise attenuation and signaldetail preserving characteristics, and outperform well-known techniques, in terms of both objective (Table 1) and subjective (Fig.3) comparisons.

\section{CONCLUSION}

In this paper, a generalized vector signal processing framework based on the order-statistic theory was presented. A robust approach for weight adaptation was tested using noisy color images. The presented WVF filters are able 
Table 1. Comparison of the presented algorithms using image Parrots.

\begin{tabular}{crlrr}
\hline Noise & \multicolumn{2}{c}{$5 \%$ noise } & \multicolumn{2}{c}{$10 \%$ noise } \\
\hline Method/Criterion & \multicolumn{1}{c}{$M S E$} & \multicolumn{1}{l}{ NCD } & \multicolumn{1}{c}{ MSE } & \multicolumn{1}{l}{ NCD } \\
\hline Noisy & 443.6 & 0.0432 & 882.0 & 0.0857 \\
MF & 63.1 & 0.0170 & 70.0 & 0.0198 \\
VMF & 64.2 & 0.0132 & 69.6 & 0.0142 \\
BVDF & 109.0 & 0.0116 & 113.5 & 0.0127 \\
DDF & 65.3 & 0.0117 & 69.7 & 0.0128 \\
WVF (3), $p=0.5$ & 18.5 & 0.0033 & 29.8 & 0.0049 \\
WVF (5), $p=0.5$ & 51.8 & 0.0091 & 58.2 & 0.0105 \\
\hline
\end{tabular}

to achieve excellent balance between smoothing and detail preservation and outperform commonly used filtering schemes.

\section{REFERENCES}

1. J. Astola, P. Haavisto, Y. Neuvo, Vector median filters, Proceedings of the IEEE, 78, 678-689, April 1990.

2. P. E. Trahanias, D. Karakos, A. N. Venetsanopoulos, Directional processing of color images: theory and experimental results, IEEE Transactions on Image Processing, 5, 868-881, June 1996.

3. T. Viero, K. Oistamo, Y. Neuvo, Three-dimensional median related filters for color image sequence filtering, IEEE Transactions on Circuits, Systems and Video Technology, 4, 129142, April 1994.

4. K. N. Plataniotis, A. N. Venetsanopoulos, Color Image Processing and Applications. Springer Verlag, Berlin, 2000.

5. R. Lukac, K. N. Plataniotis, B. Smolka, A. N. Venetsanopoulos, Generalized selection weighted vector filters, EURASIP Journal on Applied Signal Processing: Special Issue on Nonlinear Signal and Image Processing, 2004, October 2004.

6. L. Yin, R. Yang, M. Gabbouj, Y. Neuvo, Weighted median filters: a tutorial, IEEE Transactions on Circuits and Systems -II, 43, 157-192, March 1996.

7. J. Zheng, K. P. Valavanis, J. M. Gauch, Noise removal from color images, Journal of Intelligent and Robotic Systems, 7, 257-285, 1993.

8. D. G. Karakos, P. E. Trahanias, Generalized multichannel image-filtering structure, IEEE Transactions on Image Processing, 6, 1038-1045, July 1997. 